\title{
Drug treatment for early Alzheimer's disease
}

\author{
Roger Bullock
}

Alzheimer's disease is likely to be one of the challenges for the early part of the 21st century. Better knowledge of the molecular biology, genetics and pathogenesis of the condition have led to a host of psychopharmacological compounds being developed which may help in its the treatment, while epidemiological studies have suggested that existing treatments for other chronic conditions may have an effect on the presentation of Alzheimer's disease.

\section{Treatment strategies}

With current levels of understanding, there are three areas in which intervention is possible:

(a) treatment of cognitive symptoms;

(b) slowing of the disease progression; and

(c) disease prevention.

At present, the latter is the furthest goal, but research in the other areas has been increasingly productive, culminating in the launch of new drugs to influence this illness which now affects over 600000 people in the UK.

\section{Treatment of cognitive symptoms}

\section{Cholinergic therapies}

The final common pathway of Alzheimer's disease is failure of the acetylcholine neurotransmitter system (Coyle et al, 1983). This occurs as a result of a variety of factors causing the formation of plaques and tangles which, in turn, lead to neuronal death and loss of neurotransmitter potential in the cholinergic neurones (Davies \& Maloney, 1976). It was therefore logical that most early work looked at enhancing neurotransmitter function in order to promote symptomatic relief. This has led to the development of compounds acting on the cholinergic system.

Precursor loading has been tried with choline (Ferris et al, 1991), lecithin and linopirdine (Zaczek et al, 1994). No consistent results have been produced, mainly due to difficulties crossing the blood-brain barrier, and there is no indication for the use of precursors as a treatment.

Pre-synaptic releasing compounds (compounds which facilitate the release of neurotransmitters) have shown limited and inconsistent benefit. However, this avenue is still being explored and clinical trials are investigating newer agents.

Synaptic therapies, directed mainly at the inhibition of the post-synaptic enzyme acetylcholinesterase, have proved the most successful to date, and include donepezil.

Donepezil was launched in the UK in April 1997 and is the first licensed drug in this country for Alzheimer's disease (Rogers et al, 1995, 1996). Previously, hydergine had been available for the treatment of 'senile dementia' but was rarely used because of difficulties with side-effects and doubts about efficacy. Donepezil is very simple to use once daily with few side-effects. It has therefore been seen as the first treatment for Alzheimer's disease that will come into regular use.

\section{Acetylcholinesterases and their inhibition}

These enzymes are found throughout the body in both the central and peripheral nervous system,

Roger Bullock is a consultant and manager of old age psychiatry at East Wiltshire Healthcare (Victoria Hospital, Okus Road, Swindon SN1 4JU). He is Director of the Kingshill Research Centre, a unit within the department, which is involved in all phases of development of many psychiatric drugs, especially those for dementia. His research interests include psychopharmacology and neuropsychology and the use of both in primary care. 
both autonomic and motor tissues (acetylcholinesterase) and non-neural tissues, for example, liver and plasma (butyrylcholinesterase). The more specific a compound is for acetylcholinesterase, the less likely it is to have systemic effects. In normal brain the enzyme is found in the cholinergic axon and cell body, and at the synapse. In Alzheimer's disease the acetylcholinesterase is also found in the plaques, where it binds amyloid. Inhibition of the enzyme may therefore affect disease progression.

The anticholinesterase drugs form three subgroups, according to their action on the enzyme:

(a) reversible (e.g. acridines and piperidines);

(b) pseudo-irreversible (e.g. carbamates); and

(c) irreversible (e.g. organophosphates).

Of the reversible group, the only licensed example of the acridines is tacrine, which was licensed in the UK in April 1997, at the same time as donepezil. The acridines are not specific for acetylcholinesterase, and there are problems with side-effects, the most notable of which is elevation of liver enzymes which was seen in the tacrine trials (Eagger et al, 1991). Generally they have short half-lives (two hours) meaning dosing four times a day, with concomitant compliance problems.

Donepezil is a piperidine. Their dose is titrated up to the maximum tolerated level to avoid cholinergic side-effects, which include nausea and diarrhoea, and the speed of titration is important in order to minimise these effects. Piperidines have a linear dose-response relationship. Although donepezil is recommended at $5-10 \mathrm{mg}$ per day it would seem that people who tolerate the drug well can increase their dose. This has theoretical advantages, but there are no data to support it. The half-life of donepezil is 70 hours, so it can be given once daily. It does not have active metabolites and is relatively safe. Donepezil is quite selective for acetylcholinesterase, having a low activity in non-neural tissue. Caution is advised in patients with obstructive airways disease or urinary hesitancy because of the exacerbation of both by increased cholinergic activity.

Another reversible inhibitor in development is galantamine, derived from narcissi and snowdrops. This is a tertiary amine, which acts by selective competitive inhibition and again has no active metabolites. It has a half-life of six hours and can be given twice daily.

Pseudo-irreversible drugs include the carbamates rivastigmine (Anand et al, 1996) and physostigmine. These compounds bind first to the anionic site on the cholinesterase molecule before transferring to the esterase site, thus behaving like acetylcholine itself. They are metabolised in the same way, producing an inert compound. There is little activity in peripheral tissues and non-hepatic issues.

The carbamates penetrate the blood-brain barrier well. Rivastigmine has a half-life of 10 hours so can be given twice daily. It has a high selectivity for hippocampal and cortical acetylcholinesterase. This is because, of the four subtypes of acetylcholinesterase identified (G1$\mathrm{G4)}$, rivastigmine has greater affinity for the $\mathrm{G} 1$ subtype, which is present in those areas.

Irreversible inhibitors include the organophosphates, such as metrifonate. This has been available since 1962 in the treatment of schistosomiasis, and its safety and tolerability have been well established. It is metabolised to dichlorvos, a powerful but non-specific acetylcholinesterase inhibitor. The inhibition is long, recovery from the effects of dichlorvos taking 52 days while the enzyme is re-synthesised.

It is not known whether this classification has any clinical relevance. Trials show $40 \%$ of patients do well on the acetylcholinesterase inhibitors, but it is not yet clear how to predict which patients will respond, whether the same patients respond to all the compounds in a uniform way, or whether different compounds suit different people.

Post-synaptic treatment involves the use of muscarinic agonists and, more recently, nicotinic agents acting directly on post-synaptic receptors, which are generally preserved in Alzheimer's disease. Both receptor types are involved in cognition. Muscarinic agonists may also modulate beta-amyloid precursor protein. This might result in a decline in the amount of insoluble amylodogenic derivatives found in the plaques. The difficulty with the agonists to date has been a narrow therapeutic window, with frequent sideeffects and toxicity. As a result, none has a better profile than the acetylcholinesterase inhibitors. However, several compounds are now in development. The advantage of these compounds would be that while acetylcholinesterase inhibitors rely on intact neurones to produce acetylcholine, the agonists may work even after the pre-synaptic neurones have failed.

An interesting aside is that in preclinical pharmacology these agonists act well as antipsychotics in treating schizophrenia (passing all the requirements to classify as atypical antipsychotics), thus reinforcing the relationship between cholinergic and dopaminergic function. Their use in dementia and the behavioural disorders associated with it will become more relevant as their use increases. 


\section{Nicotinic therapies}

Nicotinic acetylcholine receptors are involved in learning and memory (Nordberg et al, 1989; Court \& Perry, 1994). The severity of symptoms in Alzheimer's disease has been correlated with loss in their number (Paganini-Hill et al, 1987; Aubert et al, 1992). Trials with nicotinic agonists have shown their effect on neurotransmitter levels and the up-regulation of nicotinic receptors (by reducing their turnover) (Whitehouse et al, 1986; Newhouse et al, 1988). Nicotine improves attention and information-processing, so drugs are in development. However, they also act peripherally and side-effects including hypothermia, seizures, enuresis, and cardiovascular problems, arise from stimulation of autonomic ganglia.

Nicotinic receptors can also be stimulated indirectly by galantamine (an acetylcholinesterase inhibitor discussed above), suggesting that this may have a useful dual action.

\section{Non-cholinergic therapies}

Although the cholinergic system is the one most implicated in the Alzheimer's disease process, other systems are also affected, and may offer treatment options, although the evidence is inconclusive. Monoamine oxidase inhibitor (MAOI) activity is increased in Alzheimer's disease (Strolin Benedetti \& Dostert, 1989), probably due to glial function. The MAOI, selegeline, produces an improvement in cognition (Sano et al, 1997), but this may be through improved mood. The finding has been replicated with moclobemide (Roth et al, 1996) and further trials continue with these and other drugs (Tariot et al, 1987), such as lazabemide.

\section{Slowing disease progression}

Two opportunities exist to delay progression of the illness: blocking of non-neural mechanisms which can affect neurones (e.g. microglia); and improving neural maintenance (e.g. nerve growth factor).

Propentofylline, a xanthine derivative, is a neuroprotective glial cell modulator which may be effective in both Alzheimer's disease and vascular dementia. It inhibits adenosine reuptake and also phosphodiesterase. It was first studied in ischaemic damage of the brain (Huber et al, 1993), but over 1000 patients with Alzheimer's have been given the drug with promising results. It is currently in a long-term study of disease progression. Recent data also suggest that propentofylline sensitises the muscarinic receptor. This may mean that lower doses of cholinesterase inhibitors could be given with it, thus reducing their side-effects. Like other xanthine derivatives (for example caffeine) it produces gastro-intestinal side-effects, especially as it has to be taken on an empty stomach. It also increases cerebral blood flow so dizziness and flushes can occur. It is otherwise safe and tolerated well, but must be given three times daily.

Oestrogen taken after the menopause seems to be neuroprotective (Paganini-Hill \& Henderson, 1994; Robinson et al, 1994). Women who take hormone replacement therapy have an unexpectedly low incidence of Alzheimer's disease, and in women who do get Alzheimer's disease on oestrogen therapy, the symptoms and deterioration appear less (Fillit et al, 1986). The mechanism appears to be fourfold. Oestrogen improves cerebral blood flow and stimulates nerve cell growth in the hippocampus (Birge, 1997). Because of this it boosts the production of acetylcholine, and it seems to impede the deposition of amyloid (Honjo et al, 1995). However, most of the studies to date have been observational. Randomised controlled trials are underway of efficacy, long-term prophylaxis and treatment after presentation. Oestrogen therapy is not without its dangers, increasing the risk of both breast and uterine cancer, and, of course, can not generally be used in men without a very strong indication. It is thought to be protective to the heart and skeletal system, so at present should continue to be used for these indications. Nonfeminising analogues of oestrogen, which would be available to both men and women, are being developed.

Inflammation of the brain contributes to the development of plaques and tangles. Nonsteroidal anti-inflammatory drugs (NSAIDs) appeared useful after the observation that people with rheumatoid arthritis (and, more recently, leprosy) have a low incidence of Alzheimer's disease. Activated microglia, high levels of complement and seemingly abnormal feedback of the cytokine system all point towards pathological processes which may account for nerve cell death. Three studies suggest that NSAIDs are effective both in neuroprotective and treatment terms, and clinical trials are underway (Rogers et al, 1993; Stewart et al, 1997). Many of the over-the-counter NSAIDs show this effect, except aspirin (which showed a non-significant trend to effect). NSAIDs have drawbacks in that they carry a significant 
risk of stomach distress and gastro-intestinal bleeding. Newer compounds carry a decreased risk.

Antioxidant nutrients such as vitamin $\mathrm{E}$ prevent oxidative damage. Free radicals are formed more commonly with age and eliminated less well. They have been implicated in heart disease and cancer, and there is evidence that they may have a role in Alzheimer's disease. Apolipoprotein $\mathrm{E}$ is involved in cholesterol transport. People with the ApoE4 allele have a higher risk of Alzheimer's disease. They also will have high levels of low-density lipoprotein, which favours deposition of amyloid in brain capillaries, promoting the release of free radicals. The brain is poorly provided for with antioxidants and is thus susceptible to further damage. Vitamin $\mathrm{E}$ has been given both by itself and in combination with selegeline in controlled fashion in Alzheimer's disease. The results were inconclusive, again not providing evidence of a further treatment option. However, in the USA it may be recommended that vitamin $E$ be used as an adjunctive therapy. It is certainly safe, well tolerated and inexpensive.

Nerve growth factor is necessary to maintain the cholinergic fibres in the brain. It does not cross the blood-brain barrier but can be placed by cannula into the ventricles. Clearly, this is not the most convenient treatment, but it has shown some encouraging results. New work has studied the active domains of the nerve growth factor molecule to see whether it can be engineered to cross the blood-brain barrier.

Tissue implants have been attempted with mixed success. Work continues but it is not a treatment option that looks close.

\section{Disease prevention}

While there is little available at present in this area, two areas of development offer clues.

There are at least three well-controlled studies showing that protecting against cerebrovascular disease by treating blood pressure, losing weight and stopping smoking also decreases the incidence of Alzheimer's disease, and this needs to be encouraged in anyone with vascular risk factors, especially those with early cognitive failure.

Several genes have been identified in Alzheimer's disease and some of their products isolated. Nearly all these genes are relevant in early-onset dementia's, but the prospect of gene therapy is still some way off.

\section{Principles of treatment with anticholinesterase inhibitors}

\section{Clinical benefits}

The maximum effect occurs at 12-18 weeks. The benefits seen can be divided into two areas, cognitive and functional (Schneider \& Farlow, 1995; Knopman et al, 1996).

Cognitive benefits (Cummings et al, 1987) include improvement in language skills and conversation. Orientation and ability to perform simple tasks show benefit, with increased ability to remember dates and names and items are misplaced less. In some, memory will improve, although clinically only a little, but combined with improvements in concentration and alertness the picture seems more positive to onlookers.

Functional improvements include better relating to the environment and other people (Cummings \& Victoroff, 1990). Activities of daily living show an improvement, especially in areas using common household items, self-care especially seeming to benefit. Apathy (Burns et al, 1990)(a symptom which carers find particularly difficult) is less marked on treatment, and this is associated with a concomitant mood elevation. Agitation and paranoia also improve in patients who respond to drug treatment (Gorman et al, 1993; Colenda, 1995; Cummings \& Kaufer,1996; Kaufer et al, 1996). The global picture is that patients 'get going' better and are less stubborn.

These effects will not occur in all the patients treated: $40 \%$ will show cognitive enhancement. However, clinical reports suggest up to $80 \%$ may exhibit functional improvements. If this is the case, then the use of these compounds for specific symptoms in a more structured way should be encouraged. More controlled studies are required. From the carer's perspective, functional improvement is what they are most interested in seeing.

This symptomatic improvement continues only while the drug is present. This is why early diagnosis of Alzheimer's disease is now so important, as symptomatic relief early on in the disease is clearly more valuable both to the patient and to the carer. The long-term effects of this intervention are not known, but there is controlled evidence from long-term tacrine follow-up that carers call on less primary care time and the time of institutionalisation is delayed (Lubeck et al, 1994; Knopman et al, 1996). 


\section{Confounding factors in assessing clinical response}

Four factors commonly confound assessment of clinical response to anticholinesterase inhibitors (Box 1).

\section{Unrealistic expectation}

This is the main problem at present, on the part of the carer, the patient and the doctor. Drug effects are small and subtle. Both the carer and the doctor prescribing should understand that there is not likely to be a marked improvement, a total amelioration of all symptoms or a halt to progression. Setting expectations is therefore an important part of the treatment process. In doing so, the points raised in Box 2 should be borne in mind.

Unless the carer and/or the patient understand the nature of the illness and treatment, the benefits of the pharmaceutical intervention will be lessened. For this reason, early diagnosis and information strategies are needed as part of the whole dementia programme offered locally in order to educate all those involved in the use of the drugs. This applies to the professional staff especially, as uncertainty on their part can have a profound effect on the final outcome.

\section{Failure to assess properly}

The drugs are currently only for dementia of the Alzheimer's type. A proper diagnosis must therefore be made and the stage and severity of the illness should be documented in order to assess change. Many clinicians do not use formal scales or are unfamiliar and not comfortable with the idea of using them. If detailed notes are used, they need to be taken in a systematic way, with some clear anchor points to detect what will be subtle effects.

Clinical trials with anticholinesterase inhibitors used sensitive scales to measure change. In clinical practice these may be time-consuming and unwieldy. The two most commonly used cognitive

Box 1. Confounding factors in assessing patients' response to cholinergenic therapy:

Unrealistic expectation

Failure to assess properly

Heterogeneous nature of the condition Effect of the carer
Box 2. Expectations of treatment with anticholinesterase inhibitors

The life expectancy of those with a Mini-Mental State score of $>\mathbf{2 4}$ is $\mathbf{8 - 1 2}$ years, whereas that of those with a score $<18$ is $3-4$ years.

The treatment is symptomatic, not curative. The disease will progress to a point where treatment no longer helps. Discontinuation at any stage will lead to a decline in the overall state to a point equivalent to where the patient would have been on an unmodified course.

The drugs do not work for everybody.

There may be a delay in the need for institutional care.

scales used were the Mini-Mental State Examination (MMSE; Folstein et al, 1975) and the Alzheimer's Disease Assessment Scale (ADAS; Mohs \& Cohen, 1988).The MMSE is commonly used in the UK. It is scored out of 30 points and takes about 10 minutes to administer. From the clinical trial data it can be predicted that a decline of 2-4 points per year will occur in unmodified Alzheimer's disease. The ADAS takes longer, around 45 minutes (Mohs, 1995). It is a 70-point scale, with higher scores indicating greater morbidity. This has a predicted rise of 6-7 points a year. Its length may preclude it from use in a busy clinical setting. Either of these scales can be used to measure the cognitive aspects of the response (Stern et al, 1994; Doraiswamy et al, 1997).

The cholinergic drugs also affect behavioural symptoms and the activities of daily living. Again, careful documentation can illustrate this effect, either by systematic note-taking or simple scales such as the Neuropsychiatric Inventory for behavioural symptoms (Cummings et al, 1994) and the Blessed Dementia Scale for activities of daily living (Erkinjuntti et al, 1988) .

Clinical trials also use a global clinical impression as an end-point. For research purposes this is a structured assessment, but most doctors have been trained to make global impressions and a simple record of this (e.g. minimally, moderately or markedly better or worse, or no change) gives valuable additional information.

In gauging a response to treatment, three factors are looked for, any one of which is considered a success: sustained and noticeable improvement; lack of decline; and slowing of decline. 
The method used to assess change does not matter, provided a method exists when the drug is prescribed. Not all patients respond to the compounds, so prolonged prescribing to nonresponders is costly and inefficient.

\section{Heterogeneous nature of the condition}

There is a lot of variability and fluctuation, both between patients with the same illness and in the course of the same patients' illness. Assessment is difficult if a review occurs at an unrepresentative time, for example if the patient is having a particularly good or bad spell. This can be countered in part by looking at the non-cognitive factors over the preceding month to make a judgment about the current position, although fluctuations in cognitive assessment may remain difficult to interpret.

Another difficulty in using global assessment is what to interpret if one domain improves greatly, while another significantly declines. This is a good reason for clarifying intended outcomes with the carer and patient, to refer back to if required.

Finally, because of the nature of the illness, the patient's social circumstances will alter as it progresses. Extra care at home, day centres and other social manipulation can influence the performance of the patient at a review and also affect what they are reported as being able to do. It is often easier to do something for a person than to show and encourage them how to do it for themselves. De-skilling thus becomes a problem if carers are not encouraged to help maintain the patient's maximum independence, and this will be reflected in the review.

\section{Effect of the carer}

A lot of information in the assessment process comes from the carer. Their relationship with the

Box 3. Practical issues in intiating treatment

Interview the patient and the care-giver

Establish an accurate ICD-10 diagnosis

Counsel both patient and care-giver about expectations

Establish overall treatment plan for the patient including identification of the keyworker

Measure baseline cognitive function, activities of daily living and behavioural disorders

Agree outcomes for the treatment with the care-giver and the patient

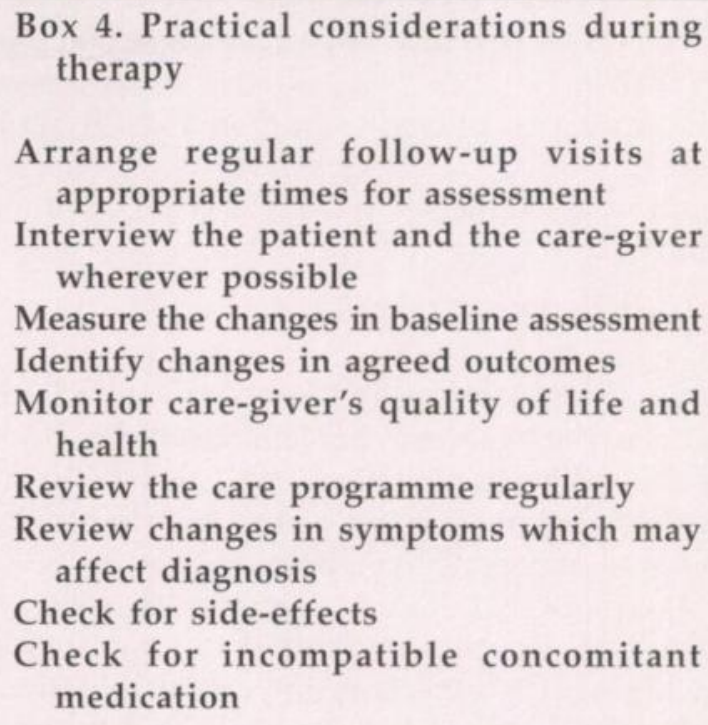

Box 4. Practical considerations during therapy

Arrange regular follow-up visits at appropriate times for assessment

Interview the patient and the care-giver wherever possible

Measure the changes in baseline assessment

Identify changes in agreed outcomes

Monitor care-giver's quality of life and health

Review the care programme regularly

Review changes in symptoms which may affect diagnosis

Check for side-effects

Check for incompatible concomitant medication

patient will colour the information obtained, as may any disproportionate event occurring in the time leading up to the review. The relationship between the carer and the person obtaining the information is also important. This is why seeing the same person, preferably at around the same time, is recommended, as is the development of good key-working processes, with families getting to know one member of the team well. This adds to the accuracy of the global knowledge of the patient's condition and the carer's quality of life.

\section{Treatment algorithm for donepezil}

In order to use treatments properly, mechanisms to get the right treatment to the right person at the right time need to be in place (see Box 3 ) along with some process to evaluate the outcome (see Box 4). A simple algorithm is useful, not only to assess progress, but also to decide when to discontinue if there is no response or if deterioration occurs.

\section{Step 1}

Identify mild to moderate dementia of Alzheimer's type. This needs accurate clinical judgement, and should be done to at least ICD-10 standards (World Health Organization, 1992). This covers an MMSE score of 10-26 (but exceptions at each end may apply).

\section{Step 2}

Make an accurate assessment of the deficits in activities of daily living and any behavioural 
disorders which may exist. Discuss with the carer all aspects of the illness, especially those that most affect them.

Produce a list of target symptoms which can be used to monitor response. Carry out a baseline cognitive assessment - the MMSE is the minimum standard.

\section{Step 3}

Discuss expectation and side-effects and the nature of the treatment. Explain the need to titrate the dose. When both doctor and patient/carer are satisfied that all questions have been answered, prescribe donepezil $5 \mathrm{mg}$ once daily.

Make sure that there is a process whereby any side-effects or other difficulties can be reported quickly. This is especially important in patients with concomitant chronic airways disease or history of peptic ulceration.

\section{Step 4}

Review the patient at four weeks, and titrate the dose up to its maximum if there have been no sideeffects, that is increase the dose to $10 \mathrm{mg}$ once daily.

\section{Step 5}

Review at 12 weeks to see whether there has been any response to medication. This is done by reevaluating any tests performed at baseline and looking specifically with the carer at the target symptoms. If there has been a noticeable and sustained response, then continue treatment and monitor at three-monthly intervals. If there was no noticeable response, then continue treatment and review at 18 weeks.

It could be argued that increasing the dose to $15 \mathrm{mg}$ in patients tolerating the drug may improve effect at this point. This is a difficult area as no data support this, but the pharmacology does. However, it is not on the data sheet and so is not a recommended route to take at present.

\section{Step 6}

At 18 weeks if, after testing, there is a noticeable and sustained response, treatment should be continued and monitored every three months. If no response is seen, then persevere up to 26 weeks and then review.

\section{Step 7}

Non-response or serious decline at 26 weeks probably indicates that the drug will not have an effect. The medication should be discontinued, after discussion with the carer and warning of possible rapid decline even in the absence of positive effect. Regular review should occur over the next four weeks to manage any such decline. Should the deterioration be too rapid or symptoms re-occur that may have been partially responding to the drug, then this may be an indication for reinstatement of therapy.

When donepezil was launched in the USA, many people switched to it from tacrine. No serious events were reported. This suggests that the compounds are interchangeable (although metrifonate has a 52-day half-life), but further controlled work is needed. It is known that cholinesterase inhibition reaches maximum clinical effect by this time in most patients.

\section{Step 8}

Long-term follow-up for responders should be at three-monthly intervals. The literature suggests that symptomatic relief lasts $6-8$ months, but there are some reports of delay in progression for 2-3 years.

\section{Implications}

These drugs will potentially have many effects, not just pharmacological, but also psychosocial and financial. Cholinergic therapies do more than enhance memory. Health economic studies imply they could in fact be self-financing if allowed to be used as part of existing well-ordered services.

\section{References}

Anand, R., et al (1996) Efficacy and safety results of the early phase studies with Exelon (ENA-713) in Alzheimer's disease: an overview. Journal of Drug Development in Clinical Practice, $8,114$.

Aubert, I., Araujo, D. M., Cecyre, D., et al (1992) Comparative alterations of nicotinic and muscarinic binding sites in Alzheimer and Parkinson diseases. Journal of Neurochemistry, $58,529-541$.

Birge, S. J. (1997) The role of oestrogen in the treatment of Alzheimer's disease. Neurology, 48 (suppl. 5), 36-41.

Burns, A., Jacoby, R. \& Levy, R. (1990) Psychiatric phenomena in Alzheimer's disease. III: Disorders of mood. British Journal of Psychiatry, 157, 81-85.

Colenda, C. C. (1995) III. Agitation: a conceptual overview. In Behavioural Complications in Alzheimer's Disease (ed B. A. Lawlor), pp. 3-17. Washington, DC: American Psychiatric Press.

Court, J.A. \& Perry, E. K. (1994) CNS nicotinic receptors. Possible therapeutic targets in neurodegenerative disorders. CNS Drugs, 2, 216-233.

Coyle, J. T., Price, D. L. \& De Long, M. R.(1983) Alzheimer's disease: a disorder of cortical cholinergenic innervation. Science, 219, 1184-1190. 
Cummings, J. L. , Miller, B., Hill, M.A., et al (1987) Neuropsychiatric aspects of multi-infarct dementia and dementia of the Alzheimer type. Archives of Neurology, 44, 389-393.

— \& Victoroff, J. I. (1990) Noncognitive neuropsychiatric syndromes in Alzheimer's disease. Neuropsychiatry, Neuropsychology and Behavioural Neurology, 3, 140-158.

-, Mega, M., Gray, K., et al (1994) The Neuropsychiatric Inventory: Comprehensive assessment of psychopathology in dementia. Neurology, 44, 2308-2314

— \& Kaufer, D. (1996) Neuropsychiatric aspects of Alzheimer's disease: the cholinergenic hypothesis revisited. Neurology, 47, 876-883.

Davies, P. \& Maloney, A. J. F. (1976) Selective loss of central cholinergenic neurons in Alzheimer's disease. Lancet, ii, 1403.

Doraiswamy, P. M., Beiber, F., Kaiser, L., et al (1997) The Alzheimer's Disease Assessment Scale. Patterns and predictors of cognitive performance in multicenter Alzheimer's disease trials. Neurology, 48, 1511-1517.

Eagger, S. A., Levy, R. \& Sahakian, B. J. (1991) Tacrine in Alzheimer's disease. Lancet, 337, 989-992.

Erkinjuntti, T., Hokkanen, L., Sulkava, R., et al (1988) The Blessed Dementia Scale as a screening test for dementia. International Journal of Geriatric Psychiatry, 3, 267-273.

Ferris, S. H., Reisberg, B. \& Crook, T. (1991) Pharmacologic treatment of senile dementia: choline, L-dopa, priacetam and choline plus priacetam. In Aging (ed S. Corkin), pp. 475-481. New York: Raven Press.

Fillit, H., Weinreb, H., Cholst, I., et al (1986) Observations in a preliminary open trial of estradiol therapy for senile dementia Alzheimer's type. Psychoneuroendocrinology, 11, 337-345.

Folstein, M. F., Folstein, S. E. \& McHugh, P. R. (1975) 'Mini-Mental State' A practical method for grading the cognitive state of patients for the clinician. Journal of Psychiatric Research, 12, 189-198

Gorman, D. G., Read, S. \& Cummings, J. L. (1993) Cholinergic therapy of behavioral disturbances in Alzheimer's disease. Neuropsychiatry, Neuropsychology and Behavioural Neurology, 6, 229-234.

Honjo, H., Tanaka, K., Kashiwagi, T., et al (1995) Senile dementia - Alzheimer's type and estrogen. Hormone and Metabolic Research, 27, 204-207.

Huber, M., Kittner, B., Hojer, C., et al (1993) Effects of propentofylline on regional cerebral glucose metabolism in acute ischemic stroke. Journal of Cerebral Blood Flow and Metabolism, 13, 526-530.

Kaufer, D. I., Cummings, J. L. \& Christine, D. (1996) Effect of tacrine on behavioural symptoms in Alzheimer's disease: an open label study. Journal of Geriatric Psychiatry and Neurology, 9, 1-6.

Knopman, D., Schneider, L., Davis, K., et al (1996) Long-term tacrine (Cognex $(\mathrm{tm}))$ treatment: effects on nursing home placement and mortality. Neurology, 47, 166-177.

Lubeck, D. P., Mazonson, P. D. \& Bowe, T. (1994) Potential effect of tacrine on expenditures for Alzheimer's disease. Medical Interface, 7, 132-138.

Mega, M., Cummings, J. L., Fiorello, T., et al (1996) The spectrum of behavioural changes in Alzheimer's disease. Neurology, 46, 130-135.

Mendez, M. F., Martin, R. J., Smyth, K. A., et al (1993) Psychiatric symptoms associated with Alzheimer's disease. Journal of Neuropsychiatry and Clinical Neurosciences, 2, 28-33.

Mohs, R. C. (1995) Neuropsychological assessment of patient's with Alzheimer's disease. In Psychopharmacology: The Fourth Generation of Progress (eds F. E. Bloom \& D. J. Kupfer), pp. 13771388. New York: Raven Press.

— \& Cohen, L. (1988) Alzheimer's disease assessment scale (ADAS). Psychopharmacology Bulletin, 24, 627-628.

Newhouse, P. A., Sunderland, T., Tariot, P. N., et al (1988) Intravenous nicotine in Alzheimer's disease: a pilot study. Psychopharmacology, 95, 171-175.

Nordberg, A., Nilsson-Hakansson, L., Adem, A., et al (1989) The role of nicotinic receptors in the pathophysiology of Alzheimer's disease. Progress in Brain Research, 79, 353-362.

Paganini-Hill, A. \& Henderson, V. W. (1994) Estrogen deficiency and risk of Alzheimer's disease in women. American Journal of Epidemiology, 140, 256-261.

Perry, E. K., Perry, R. H., Smith, C. J., et al (1987) Nicotinic receptor abnormalities in Alzheimer's and Parkinson's diseases. Journal of Neurology, Neurosurgery and Psychiatry, 50, 806-809.
Robinson, D., Friedman, L., Marcus, R., et al (1994) Estrogen replacement therapy and memory in older women. Journal of American Geriatric Society, 42, 919-922.

Rogers, J., Kirby, L. C., Hempelman, S. R., et al (1993) Clinical trial of indomethacin in Alzheimer's disease. Neurology, 43, 1609-1611.

—, Perdomo, C. \& Friedhoff, L. T. (1995) Clinical benefits are maintained during long-term treatment of Alzheimer's disease with the acetylcholinesterase inhibitor, E2020. European Neuropsychopharmacology, 5, 386.

- Friedhoff, L. T., \& The Donepezil Study Group (1996) The efficacy and safety of donepezil in patients with Alzheimer's disease. Results of a US multicenter randomised double blind placebo controlled trial. Dementia, 7, 293-303.

Roth, M., Mountjoy, C. Q. \& Amrein, R. (1996) Moclobemide in elderly patients with cognitive decline and depression: An international double-blind, placebo-controlled trial. British Journal of Psychiatry, 168, 149-157.

Sano, M., Ernesto, C., Thomas, R. G.,et al (1997) A controlled trial of selegeline, alpha tocopheral, or both, as treatment for Alzheimer's disease. New England Journal of Medicine, 336, 1216-1222.

Schneider, L. S. \& Farlow, M. R. (1995) Predicting response to cholinesterase inhibitors in Alzheimer's disease: possible approaches. CNS Drugs, 4, 114-124.

Stern, R. G., Mohs, R. C., Davidson, M., et al (1994) A longitudinal study of Alzheimer's disease: measurement, rate and predictors of cognitive deterioration. American Journal of Psychiatry, 151, 390-396.

Stewart, W. F., Kawas, C., Corrada, M., et al (1997) Risk of Alzheimer's disease and duration of NSAID use. Neurology, 48, 626-632.

Strolin Benedetti, M. \& Dostert, P. (1989) Monoamine oxidase, brain ageing and degenerative diseases. Biochemical Pharmacology, 38, 555-561.

Tariot, P. N., Cohen, R. M., Sunderland, T., et al (1987) L-deprenyl in Alzheimer's disease. Preliminary evidence for behavioural change with monoamine oxidase B inhibition. Archives of General Psychiatry, 44, 427-433.

Whitehouse, P. J., Martino, A. M., Antuono, P. G., et al (1986) Nicotinic acetylcholine binding sites in Alzheimer's disease. Brain Research, 371, 146-151.

World Health Organization (1992) The Tenth Revision of the International Classification of Diseases and Related Health Problems. (ICD-10). Geneva: WHO.

Zaczek, R., Chorvat, R. J., Earl, R. A., et al (1994) Neurotransmitter release enhancement as a possible therapy for neurodegenerative diseases: update on linopirdine (DUP996). In Alzheimer Disease: Therapeutic Strategies (eds E. Giacobini \& R. Becker), pp. 252-255. Boston, MA: Birkhauser.

\section{Multiple choice questions}

1. The following are examples of reversible anticholinesterase inhibitors:
a piperidines
b carbamates
c phenothiazines
d acridines
e organophosphates.

2. Which of the following provide potential treatment options in Alzheimer's disease:

a serotonergic therapies

b monoamine oxidase inhibitors

c muscarinic agonists

d dopaminergic therapies

e non-steroidal anti-inflammatory drugs? 
3. Which of the following confound the clinician's assessment of change when assessing a patient on cholinergic therapy:

a relationship with care-giver

b false expectation of the carer

c fluctuation in the illness

d use of complex measurements

e disproportionate events prior to the assessment?

4. Evidence shows improvement in patients on anticholinesterase treatment in:
a psychotic symptoms
b agitation
c apathy
d conversational skills
e performance in household tasks.

5. Discontinuation of donepezil:

a will lead to a deterioration of the patient to where they would have been at this stage if untreated

b should be considered if there has been no response to treatment after six weeks c should be considered if the dementia has declined such that the benefit is not apparent and other treatments (e.g. antipsychotics) are necessary

d needs to be a joint decision between doctor, carer and user

e will lead to an immediate reduction in cholinergic side-effects.

\begin{tabular}{|c|c|c|c|c|c|}
\hline \multicolumn{6}{|c|}{ MCQ answers } \\
\hline 1 & 2 & 3 & 4 & & 5 \\
\hline a $\mathrm{T}$ & a $F$ & a $T$ & a & $\mathrm{T}$ & a $\mathrm{T}$ \\
\hline b F & b $\mathrm{T}$ & b $T$ & b & $\mathrm{T}$ & b $F$ \\
\hline c $F$ & c $\mathrm{T}$ & c $\mathrm{T}$ & c & $T$ & c $\mathrm{T}$ \\
\hline d $T$ & d F & d F & d & $T$ & d T \\
\hline e $F$ & e $T$ & e $T$ & e & $T$ & e $F$ \\
\hline
\end{tabular}

